\title{
Mitteilungen für die Generalversammlung am 25. April 1914.
}

(l. Geschäftsbericht, II. Netto-Bilanz, III. Vorschläge für die Vorstandswahlen.)

\section{Geschäftsbericht}

\section{des Vorstandes über das Jahr 1913.}

Folgende Tabelle zeigt den Mitgliederbestand des Jahres 1913 im Vergleich mit dem der vorhergebenden Jabre.

\begin{tabular}{|c|c|c|c|c|c|c|c|}
\hline & $\begin{array}{c}1907 \\
(\operatorname{am} 1: 1 . \\
1908)\end{array}$ & $\begin{array}{c}1908 \\
(\mathrm{~nm} 1.1 \\
1909)\end{array}$ & $\begin{array}{c}1909 \\
(\operatorname{am} 1.1 \\
1910)\end{array}$ & $\begin{array}{c}1910 \\
(\operatorname{am} 1.1 \\
1911)\end{array}$ & $\begin{array}{c}1911 \\
(\operatorname{am~} 1.1 \\
1912)\end{array}$ & $\begin{array}{c}1912 \\
(\operatorname{an} 1.1 . \\
1913)\end{array}$ & $\begin{array}{c}1913 \\
(\operatorname{am} 1.1 . \\
1914)\end{array}$ \\
\hline E.-M. & $1 j$ & 15 & 16 & 17 & 14 & 14 & 17 \\
\hline O. M. & 3034 & 2970 & 2917 & 2874 & 2968 & 3012 & 3064 \\
\hline \multirow{2}{*}{ A. M. } & 275 & 251 & 263 & 246 & 370 & 330 & 312 \\
\hline & 3324 & $3: 36$ & 3196 & 3137 & 3352 & 3356 & 3393 \\
\hline
\end{tabular}

Aus dieser Zusammenstellung ist eine Steigerung der Mitgliederzahl im abgelaufenen Jahr zu ersehen.

Besonders erfreulich ist die Beobachtung, daß seit 1911 die Zahl der ordentlicben Mitglieder beständig im Steigen begriffen ist.

Der Umfang der »Berichte 1913 sowie Anzahl und Länge der Abhandlungen ist aus folgender Zusammenstellung ersichtlich:

\begin{tabular}{|c|c|c|c|c|c|c|}
\hline Jahr & . & 1909 & 1910 & 1911 & 1912 & 1913 \\
\hline $\begin{array}{l}\text { Redaktioneller 'Til } \\
\text { (ausschl. Schlußheit) }\end{array}$ & Seiten & 4940 & 3643 & 3756 & 3779 & 4116 \\
\hline obne Nekrologe. . . . . & $»$ & & & 3512 & 3602 & 4052 \\
\hline Zahl der Abhandlungen & . & 717 & 559 & 500 & 496 & $5 \pm 3$ \\
\hline Durehschn. Umf. d. Ahhandlg. S & Seiten . & 6.8 & $6 . j$ & 7.0 & 7.2 & 7.7 \\
\hline
\end{tabular}

Im Jahre 1913 sind von $27 \mathrm{Nichtmitgliedern} \mathrm{Abhandlungen} \mathrm{einge-}$ gangen. Der Umfang der von Nichtmitgliedern aufgenommenen Abhandlungen beträgt 152 Seiten. 7 dieser Nichtmitglieder sind der Gescllschaft im Jahre 191? beigetreten. 
Von dem wissenschaftlichen Leiter der Abteilung für chemische Sammelliteratur, Hrn. P. Jacobson, ist der folgende Bericht eingegangen:

»Das Material, welches die unter Leitung von Hrn. Prof. A. Hesse stehende Redartion des "Chemischen Zentralblatts « im Jahre 1913 \%u verarbeiten hatte, war erheblicb größer als in den Vorjahren, wie die nachstehende Übersicht zeigt".

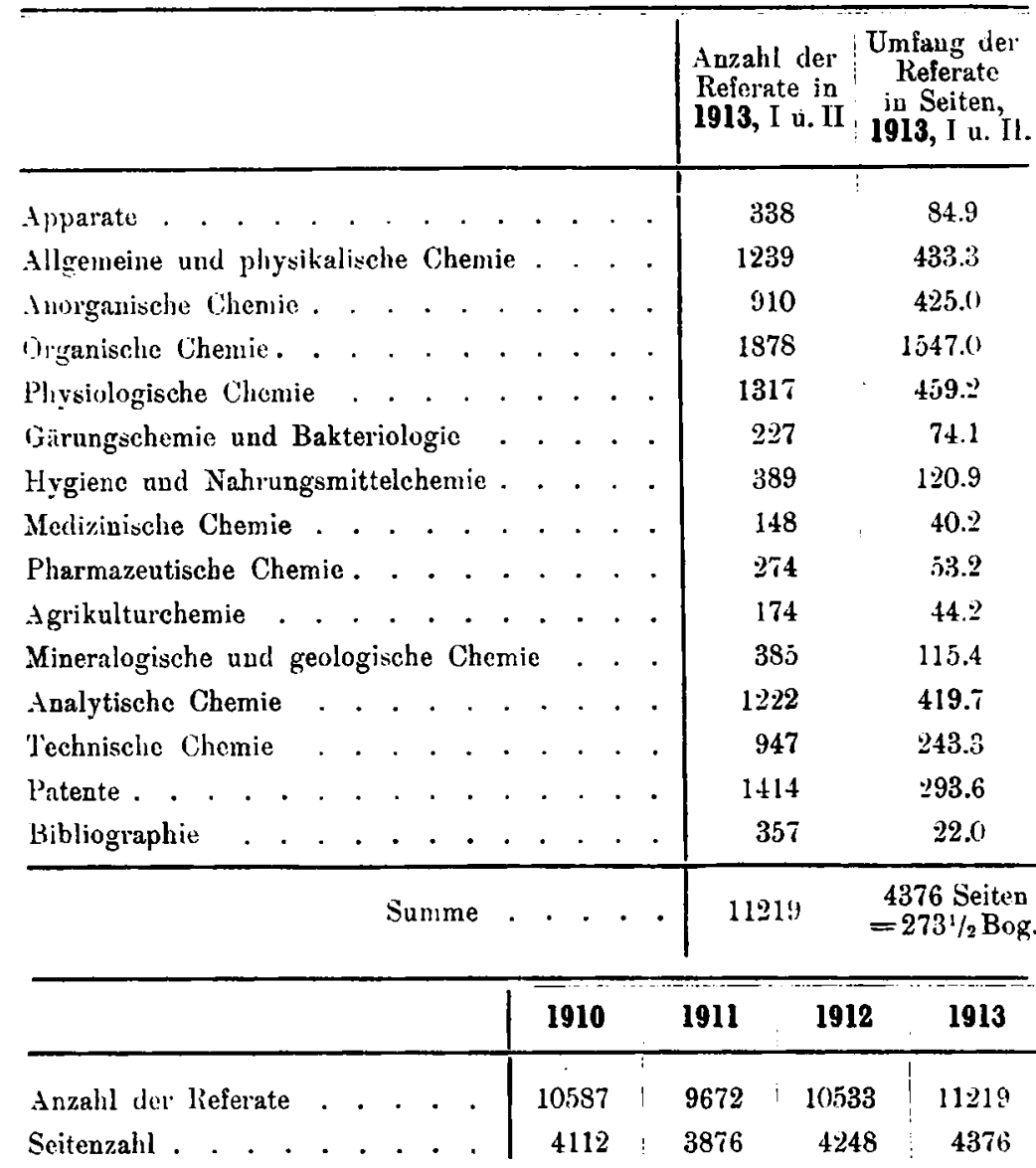

*Gegenüber dem Jahre 1912 beträgt der Zuwachs an Referatenzabl $6.5 \%$, an Seitenzahl $3.0 \%$. Es ist also gelungen, die Umfangsvermehrung auf einer niedrigeren Stufe zu halten, als die Stoffiermehrung. Dauk diesem Umstande und einer Erhöhung des buchbändlerischen Absatzes ist der diesjährige Fehlbetrag des Budgets nur um eine kleine Summe größer als der vorjährige«. 
-Die außerordentlich günstige Aufnahme, welche die Zusammenfassung des Zentrallblatt-Inhalts in fünfjährigen Generalregistern') bei den Abonnenten findet, hat zu Maßnahmen geführt, um schon jetzt die lür das Jahr 1917 in Aussicht zu nehmende Ausgabe eines vierten Generalregisters, welches die Jahrgänge 1912-1916 umfassen soll, vorzubereiten. Gleichzeitig wurde eine OrganisationsÄnderung vorgenommen, um unserem Referier-Organ den häufig gerühmten Vorzug, daß seine Halbjabrs-Register regelmäßig zuyleich mit dem letzten Hefte erscheinen, auch für die Zukunft zu erhalten. Zu diesem Zweck wird vom, Jahre 1914 ab die Bearbeitung der halbjährlichen Sachregister und des Generalregisters einem Beamten übertragen, der mit den laufenden Arbeiten für die einzelnen Hefte nichts zu tun hat. Hr. Dr. I. Bloch bebält diese Aufgabe, für die er in langjähriger 'Tätigkeit reiche Erfahrung gesammelt hat, bei, während er seine bisherigen Funktionen als stellvertretender Redakteur vom 1. Oktober 1914 ab aufgeben und in ihnen durch Hrn. Dr. E. Förster ersetzt werden wird.*

$\gg$ Der erste, von Hrn. Dr. R. Stelzner redigierte Band der

Literatur-Register der organischen Chemie, geordnet nach M. M. Richters Formelsystem,

ist im Juli des abgelaufenen Jahres zur Ausgabe gelangt. Er umfaßt die Literaturjabre 1910 und 1911, ist rund 1300 Seiten stark und enthält gegen 58000 Angaben über etwa 22000 verschiedene Substanzen. Bei der Bearbeitung des Literatur-Materials wurde der Redakteur von Hrn. Dr. G. Haas, beim Ordnen des Manuskripts für den Druck und der Überwachung der Drucklegung von Frl. Dr: H. Kuh, bei den Korrekturen und der Zusammenstellung des Trivialnamen-Registers ferner von den HHrn. Dr. E. Kindscher, Dr. R. A scher und Dr. H. Schönfeld unterstïtzt. Allen diesen Kräften, zu denen für den zweiten, alsbald in Angriff genommenen Band (1912/13) noch Frl. Dr. A. Fiedler getreten ist, gebührt wärmster Dank für die Energie, das Geschick und die Sorgfalt, mit der sie die ebenso mühevolle wie verdienstliche Aufgabe durchgeführt baben.k

„Die Druckleging dieses Bandes hat leider die Fertigstellung les $\gg$ Berichte - Registers für den Jahrgang 1912 verzögert: es konnte erst Ende September ausgegeben werden ${ }^{2}$ ). Für das nächste $\gg$ Berichte $\ll-$ Register aber wird wieder der übliche Ausgabe-'Termin eingehalten werden, so daß es im Laufe des Aprils 1914 in den Händen der Mitglieder sein wird.*

„In dem vorigen Jabresbericht ist eingebend geschildert worden ${ }^{3}$ ), welcbe Vorteile die "Literatur-Register der organischen Chemie

ग) Vergl. dazu B. 46, 636 [1913].

2) Vergl. B. 46, 2493-2494 [1913]. 3) Vergl. B. 46, 637-638 [1913]. 
durcb ibre Vollständigkeit und bequeme Handhabung den wissenschaftlich und technisch tätigen Chemikern, sowie den auf den Grenzgebieten der organiscben Chemie Arbeitenden bieten. Ihre Wichtigkeit wird noch dadurch gesteigert, da $\beta$ sie - wie aus den folgenden Darlegungen ersichtlich wircl - mit dem Beginn ibrer Literatur-Berücksichtigung an den Literatur-Scblußtermin für die vierte $A$ uflage von Beilsteins Handbuch der organischen Chemie anknüpfen.*

„Über die Vorbereitung dieses großen Unternehmens ist jährlich kurz berichtet worden ${ }^{1}$ ). Sie gliedert sich in die folgenden Etappen:

A. die Ausarbeitung eines neuen Systems (1907),

B. die Einordnung des die Literatur bis etwa 1905 umfassenden Materials an Artikeln in das neue System, verbunden mit einer kritischen Durchsicht und Reinigung des Textes der dritten Beilstein-Auflage einschließlich ihrer Ergänzungbände $(1908-1912)$,

C. die Bearbeitung der neueren Literatur.*

"Die ursprünglich nicht geplante, aber im Verlauf der Arbeit als unerläßlich erkannte, durchgreifende Textkritik der früheren Auflage hat den zweiten Teil dieser Vorarbeiteu (B) sehr viel zeitraubender gestaltet, als vorausgeseben werden konnte. Anfangs des abgelaufenen .lahres hat nun die unter Leitung von Hrn. Dr. B. Prager stehende Redaktion, welcher als ständige Mitarbeiter Hr. Dr. P. Schmidt, Frl. Dr. D. Steru und Hr. Dr. R. Czensoy, als nichtständige Hilfskraft Hr. Dr. A. Wagner angehören, den letzten Teil der Vorarbeiten (C) begonnen. Nach den Erfahrungen des Jabres 1913 ist z.11 erwarten, daß die Redaktion - falls keine Störungen eintreten - im Herbst 1915 den Literatur-Schlußtermin vom 1. Januar 1910 erreicht haben wird. Nitwa ein balbes Jahr später würde dann der Druck begonnen werden können.*

"Für die Drucklegung selbst haben nun dem Yorstand in letzter Teit zwei Pläne zur Beratung vorgelegen. Nach dem einen wäre der Literatur-Schlußtermin für die ejnzelnen Bände regelmäßig vorzuschieben, aach dem andren der erste Literatur-Scblußtermin für das ganze Werk beizubebalten. Wäbrend bei der gegenwärtigen $\mathrm{Zu}$ sammensetzung der Redaktion die notwendige Druckzeit für den ersten Fall auf ca. 14 Jahre geschätzt wurde, läßt sich für den zweiten Fall - soweit solche Schätzungen überhaupt möglich sind - die Beendigung der Drucklegung in der halben Zeit, also in ca. 7 Jahren, erhoffen."

b) Vergl. B. 40, 5028 [1907]: 41, 4489 [1908]; 42, 4923 [1909]: 43, $36 \div 6[1910] ; 45,698[1912] ; 46,639$ [1913]. 
*Der Vorstand bat sich für den zweiten Plan entschieden, besouders weil nach ihm die Interessenten rascher in den Besitz eines abgeschlossenen, alle Teile der organischen Chemie umfassenden Werkes kommen. Dieses Werk, dessen Umfang auf rund 950 Bogen (=15000 Seiten) sich schätzen läßt, wird voraussichtlich die letzte enzyklopädische Zusammenfassung der gesamten organischen Chemie vilden. Fs soll ihren Stand am SchluB des ersten Jahrzehnts des zwanzigsten Jahrhunderts aufzeichnen.*

*An den Literatur-Schlußtermin der vierten Beilstein-Auflage schließen nun die oben besprocbenen Literatur-Register der organischen Chemie « unmittelbar an. Diese Register, die in zweijährigen Perioden erscheinen sollen, werden also den künftigen Besitzern des "Beilstein* die Möglichkeit bieten, sich auf bequemste Weise dessen Text durch den Nachweis der neueren, über den Beilstein-Schlußtermin hinausgebenden Literatur lückenlos zu ergänzen. so werden sie dauernd ibren Wert bebalten, zum mindesten aber, bis etwa ein Supplement zur vierten Beilstein-Auflage abgeschlossen vorliegen sollte, was frühestens um das Jahr $1930 \mathrm{zu}$ erwarten ist.

-Daß die dritte Beilstein-Auflage mit ihrea Ergänzungs$b$ änden, von denen nur noch ein geringer Vorrat zur Verfügung steht, noch fïr eine ganze Reihe von Jahren - nämlicb bis zum Abschlu $\beta$ der vierten Auflage, also ca. 192: - für die Bearbeiter der organischen Chemie ein unentbehrlicher Ratgeber bleiben wird, wurde schon im vorjährigen Bericht hervorgehoben ').*

»Das aus den Mitteln der Beilstein-Vereinigung unterstützte Lexikon der anorganischen Verbindungen ${ }^{2}$ ) ist im letzten Jahre von seinem Begründer, Hro. Dr. M. K. Hoffmann und seiner Mitarbeiterin Frl. Dr. E. Micbel rüstig gefördert worden. Erschienen sind seit dem vorjährigeı Bericht ${ }^{3}$ ) vom Bande I, S. 81-464, $\mathrm{S}-\mathrm{Ba}$, Nr. 7-22 und rom Bande II, S. 577-1072, $\mathrm{Cr}-\mathrm{Pt}$, Nr. 63-71. Ende 1914 wird dieses Werk, welches den Bestand an anorganischen Verbindungen mit der zugehörigen Literatur bis 1910 verzeichnet, vollendet vorliegen. Es soll durch Supplemente ergänzt werden, über deren Bearbeitung gegenwärtig Beratungen schweben."

W. Will, Präвident.

1) Vergl. B. 46, 639 [1913].

2) Vergl. B. 45, 699 [1912]: 46, 639 [1913].

3) Vergl. B. 46, 639 [1913]. 
II. Netto-Bilanz nebst Gewinn- und Verlust-Konto der Deutschen Chemischen Gesellschaft per 31. Dezember 1913.

Netto = Bilanzen

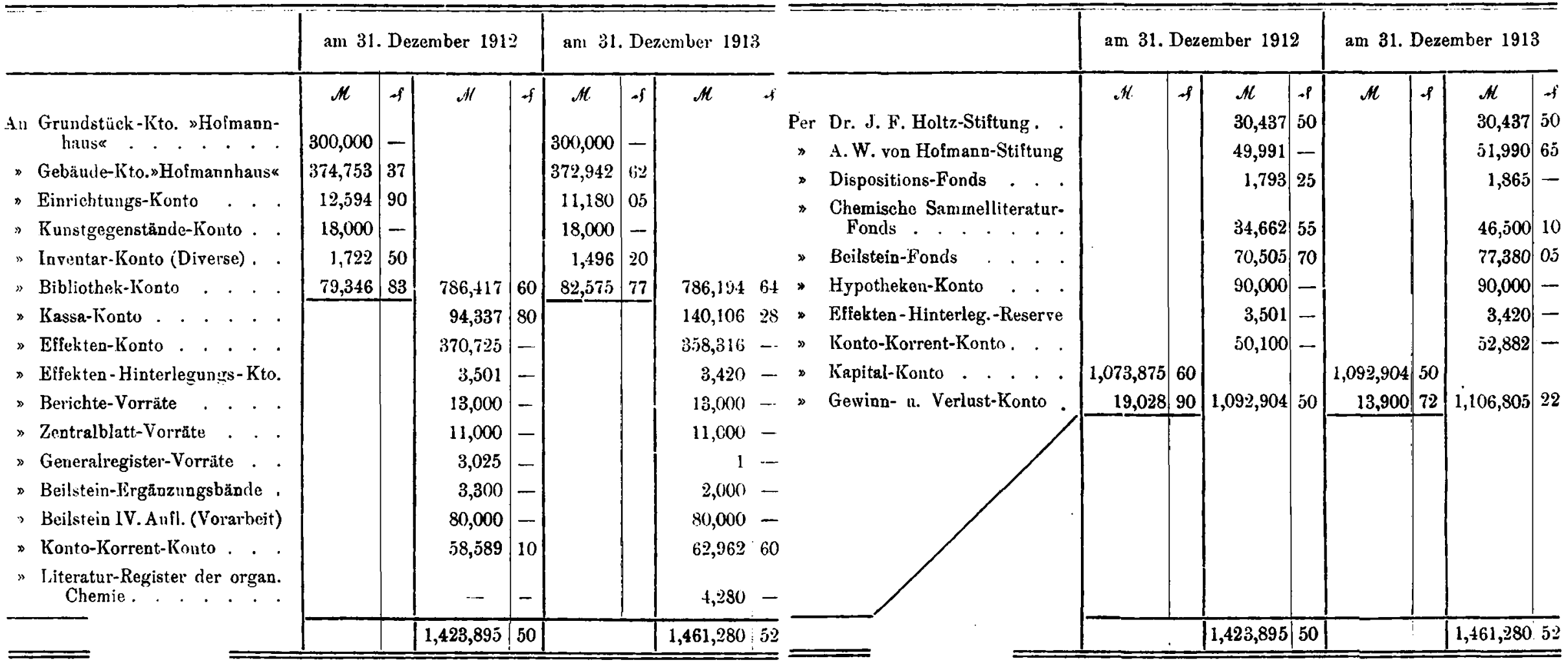

Debet

Gewinn- und Verlust-Konto

Credit

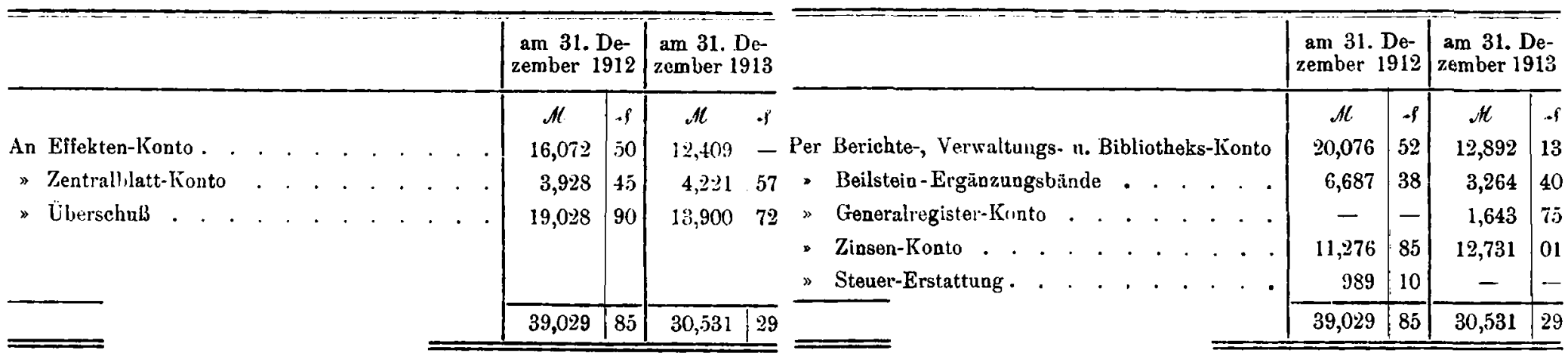


Effektive Kosten in 1913.

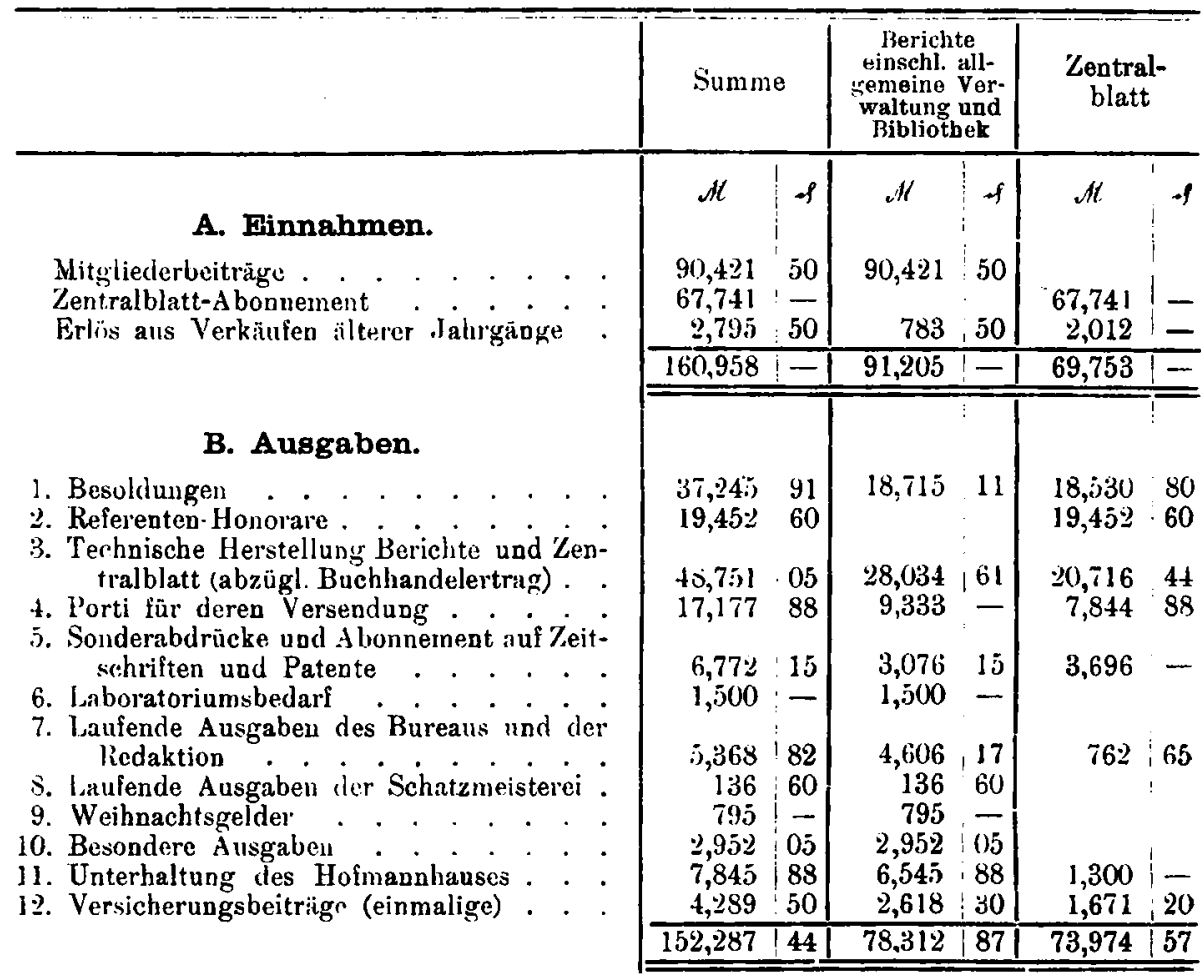

\section{Gewinn-Nachweisung.}

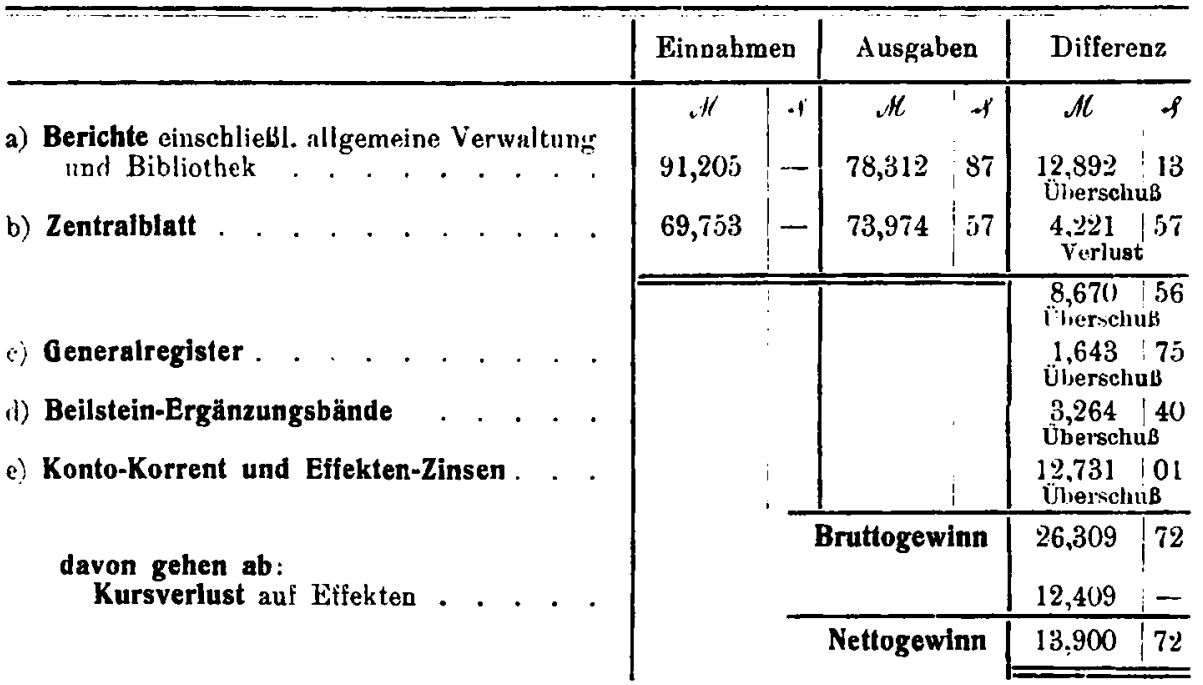


Kosten des Hofmann-Hauses.

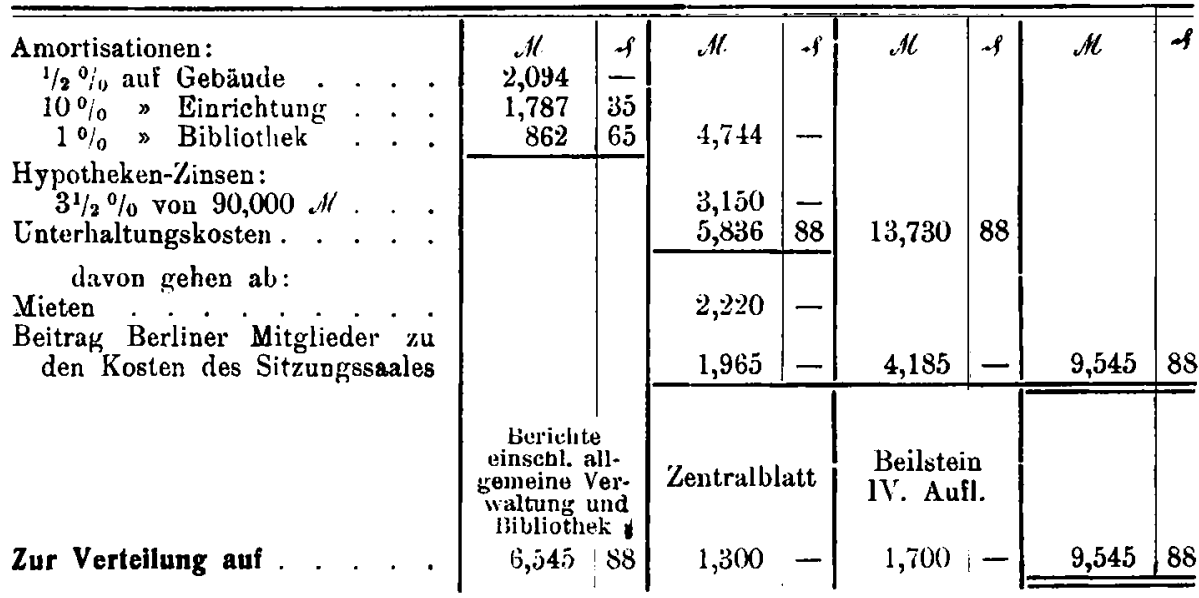

Chemische Sammelliteratur-Fonds.

Schenkung der Firma Leopold Cassella \& Co. ab Schenkungssteuer . . . . . . . . .

+ Zinsen 1910/1913

Vorarbeit 1910/13 fūr Lit.-Reg. I abz. Autoren. Honorar der Verlagsbuchhandlung (Verlust)

Differenz zwischen Zahlungen an die Buchhandlung für. Mitglieder-Exemplare und eingegangenen Zahlungen, für Jit.-Reg. I (Verlust)

Wert am 31. Dezember 1913 . . . . . .

Vorarbeit 1913 für Lit.-Reg. II . . . . . .

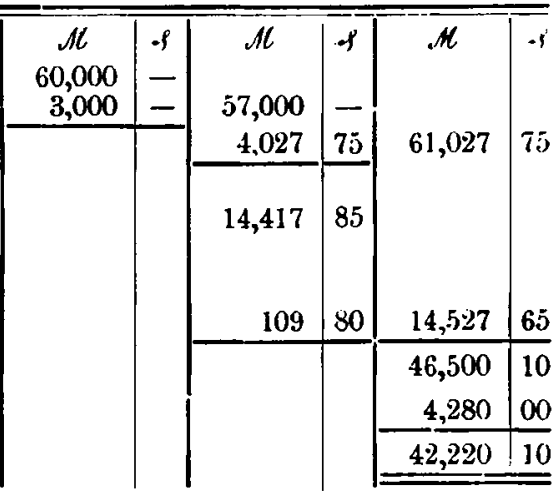

Bestand am 31. Dezember 1913 . . . . 


\section{Beilstein-Fonds.}

\section{Einnahmen:}

Stand am 1. 1. 1913

4. Rate p. 1913 der Vereinigung

1 Vorauszahlung in 1912

$$
+\quad \text { lür } 1914 \text {. }
$$

+ Zinsen p. 1913

ab Subventionierung des Hoffmann-Lexikons III. Rate

Versicherungsbeitr. (einmalige)

\section{Ausgaben :}

Besoldungen

Referenten-Honorare

LaufendeA usgaben d. Redaktion Abschreibung auf Inventar . Unterhaltung des HofmannHauses

Stand am 31.12.1913. .

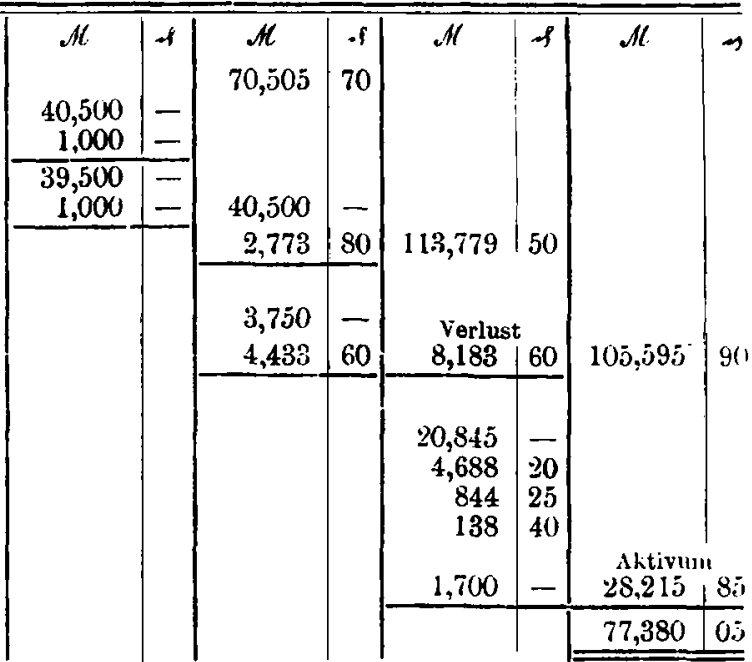




\section{Vorschlăge des Vorstandes für die Vorstands- Ergănzungswahlen.}

Als Präsident:

$$
\text { I. Knorr, (Jena), }
$$

als einheimischer Vizepräsident ( $1 \mathrm{zu}$ wählen):

H. Wicbelhaus, F. Haber, R. Willstätter, als auswärtiger Vizepräsident:

L. Gans (Frankfurt a/M.),

als Schriftführer:

$$
\text { B. Lepsius, }
$$

als stellvertretender Schriftführer:

R. Pschorr,

als Schatzmeister:

$$
\text { F. Oppenbeim, }
$$

als einheimische Ausscbubmitglieder (drei zu wäblen):

K. A. Hofmann, H. Bucherer, E. Erlenmeyer, A. Rosenbein, O. Diels, J. Meisenbeimer,

als auswärtige Ausschußmitglieder (fünf zu wählen):

R. Zsigmondy (Göttingen), A. Winther (Offenbach), H. Specketer (Griesheim), H. v. Meister (Höchst), E. A. Merck (Darmstadt), R. Scholl (Graz), H. Staudinger (Zürich), J. Stobbe (Leipzig).

Im Vorstand verbleiben statutengemäß außer den ehemaligen Präsidenten und Vizepräsidenten die HHra.: E. Beckmann und C. Duisberg als Vizepräsidenten; F. Mylius als Schriftüurer; B. Lepsius als stellvertretender Schriftführer; W. Marckwald als Bibliothekar; F. Haber, W. Traube, R. Willstätter als einheimische AusschuBmitglieder; F. Kehrmann, C. L. Müller, O. Piloty, A. Reissert, D. Vorländer als auswärtige Ausschußmitglieder. 